\title{
Cerebrovascular neuroimaging: which tests and when?
}

Michael Hoffmann

Durban Cerebrovascular Group, Department of Surgery, University of Natal, Durban

Cerebrovascular neuroimaging requires careful clinical and radiological correlation for interpretation. With a profusion of tests available, what is the best investigation and when?
D etermination of brain pathology and pathophysiology was for a long term largely deductive with no objective evidence. Early neurologists had to wait for autopsy information for a definitive diagnosis. Both the impervious, intact skull and the dearth of clinically useful brain treatment strategies contributed to the "dark ages of the brain". The situation today is vastly different in that we are overwhelmed with an ever increasing array of new investigative tools. These include colour duplex Doppler flow imaging (CDFI), transcranial Doppler (TCD), magnetic resonanceimaging (MR), magnetic resonance angiographic imaging (MRA), single photon emission computed tomographic imaging (SPECT), spiral computerised tomography (helical or spiral CT) and positron emission tomography (PET). Coincident with such a wide choice of brain imaging methods, are the imminent acute stroke therapies (antithrombotics, thrombolytics, neuroprotective and gene based therapies) that are anticipated within the next few months to years. Despite the complexity of the brain, outward manifestations of impairment remain limited to long tract signs, visual deficits and cognitive deficits, underscoring the need for paraclinical evidence of malfunction.

Colourduplex flow imaging (CDFI) has become a routine test for extra cranial arterial assessment with excellentsensitivity and specificity as compared to angiography. ${ }^{1}$ Plaque characteristics are most accurately assessed with CDFI and area stenosis as measured by Doppler as opposed to diameter stenosis as measured by angiography is a more accurate correlate of the true anatomical degree of stenosis. ${ }^{2}$ A recent and at present still clinical investigation extension of CDFI is power Doppler imaging (PDI). This new modality generates intravascular colour signals from the amplitude of the echo signal, which in turn depends on the intensity of the sampled red blood cells. It overcomes the two major limitations of CDFI which are the assessment of high grade stenosis and the intrastenotic diameter and area reduction in heavily calcified plaques, which are commonly encountered. In addition the method is angle independent and free from aliasing artifacts which is a limitation of CDFI.The PDI contrasts the lumen with colour similar to angiography. In this respect it essentially bridges the gap between angiography and CDFI whose major limitations were inability to image the outer vessel boundary and residual vessel lumen. ${ }^{3,4}$

Transcranial Doppler(TCD) has established applications for the reliable diagnosis of intracranial vessel stenosis, vasospasm, Willisian collateralisation and arteriovenous malformations. In addition, it is useful for the detection of vasomotor testing and monitoring and brain death. ${ }^{5} \mathrm{~A}$ more recent and highly clinically relevant application is in the detection of intracranial emboli, whether of cardiac, large artery or exogenous source. The diagnosis of patent foramen ovale, an important predisposing factor in a significant proportion of young stroke patients of "undetermined aetiology", is most easily and cheaply done by contrast("bubble")TCD. ${ }^{6}$ The differentiation of symptomatic from asymptomatic carotid stenoses can also be facilitated by determining the degree of artery to artery embolisation by TCD by performing HITS (high intensity transient signals) counts. ${ }^{7}$ Both duplex and powerTCD 


\section{from page 6}

are currently in the clinical investigational stage with promise for considerable widening of their applications.

Magnetic resonance imaging has afforded the excellent anatomical detail of the brain especially in areas which do notshow up well on computerised tomographic (CT) scanning such as the brainstem, cerebellum and spinal cord. Routine MR imaging by spin echo sequences, the best known being $\mathrm{Tl} 1, \mathrm{~T} 2$ and proton density weighted images. Tl images enable high tissue to tissue differentiation (anatomy), T2 images are most useful for intratissue characteristics (pathology) and proton density weighted images aid differentiation of focal tissue changes adjacent to free fluid such as the subarachnoid space and ventricles. This has resulted in unheralded accuracy of diagnoses of posterior circulation infarction especially of the brainstem, cerebellum and diencephalon. Thrombolysis - with tissue plasminogen activator is now indicated for a subset of acute stroke patients. ${ }^{8}$ The accurate, early detection of this treatable subgroup of patients is dependent on the appropriate investigative technique. As a consequence, the requirement for ultra early detection of infarctive and haemorrhagic components is of vital importance. Two new investigative techniques, $M R$ diffusion (MRD) and MR perfusion (MRP) allow tissue changes of cytotoxic oedema to be seen within 1 hour of onset. ${ }^{9}$ Ultrafast echoplanar imaging may reduce this to about 30 minutes and allowsimaging of ischaemic tissue that has been experimentally reversed in the animal model by drugs such as NMDA antagonists. ${ }^{10,11} \mathrm{Al}$ though not routine in clinical practice, these methods hold promise. MR angiography (MRA) has already reached routine clinical practice with the two principle techniques; namely, time of flight (TOF) and phase contrast (PC) each having their particular attributes. ${ }^{12}$ Most usefully combined with transcranial Doppler and duplex
Doppler to grade stenoses, a two and three dimensional cerebrovascular tree can be imaged entirely noninvasively. MRA adds only about 10 minutes to the conventional MRI scan of the brain and it is easy to see that this may rapidly become a routine part of cerebrovascular imaging. Finally, magnetic resonance spectroscopy (MRS) although not yet in clinical use, may detect the earliest possible changes of ischaemia at cellular level by measuring increases of lactate and a fall in highenergy phosphates. ${ }^{13}$

Computerised tomographic brain scanning hasimproved with respect to definition and speed of examination with each new generation of scanners. Because of its inherentdrawbacksit is relegated to the task of characterisation of sudden brain syndromes into either mass lesions or stroke, and in the case of stroke, to differentiate haemorrhage from bland infarction. However due to its popularity and relatively lower cost it will probably retain its position as the most widely used brain scanning method. The novel application of helical CT scanning of blood vessels has become so useful clinically that even dissection of carotid arteries, until recently the domain of conventional angiography, can be reliably diagnosed by this method. ${ }^{14}$

SPECT has been shown to be a sensitive indicator of cerebral perfusion and the most sensitive brain scan for the demonstration of ischaemia and infarction in the acute phase. Three different tracers are available for clinical use; HMPAO, Iodine 123 and Tc ethyl cysteinate dimer. The radionucleotide is injected intravenously and accumulates in different areas of the brain proportionate to the rate of delivery of nutrients/blood flow to that volume of brain tissue. Technology has allowed the combination of gamma cameras and computerised tomography to create three dimensional construction of images. The major advantage of SPECT is its functional imaging capacity which complements the standard anatomical imaging using magnetic resonance and the older computerised tomography scans. SPECT is also affordable and considerably cheaper than positron emission tomography (PET).The latter is globally limited by cost and the necessity for proximity to radionucleotide processing (a cyclotron). In fact, the cost of SPECT is similar to the conventional CT scan without contrast. The major advantage of PET over SPECT isimproved resolution and its capability for themeasurement of regional cerebral metabolism. Biochemical information from receptor activity measurementsuch as muscarinic, benzodiazepine, serotonergic and dopaminergic receptor systems are possible but are not in routine clinical use and remain experimental at the time of writing. ${ }^{15}$

The quest for pathophysiological subtyping in acute stroke has never been more urgent. The long list of animal-effective, human-failure stroke drugs has been blamed squarely on the unrealistically long time window of $24-48$ hours or more for patient recruitment. Now that we appreciate that "time is brain" and strive for sub 6 hour recruitment period for acute stroke intervention, this may nevertheless be unnecessarily rigid and exclude potentially salvageable patients with slightly longer time frames. ${ }^{16}$ Such subtyping can only be done with the strategic choice of a combination of functional and structural neuroimaging. By means of TCD andMRA the site of arterial occlusion can be defined in order to determine the desirability of thrombolytic therapy and to exclude patients with distal arterial or branch occlusion or those without occlusion. Tissue viability can be determined to some degree by early SPECT, diffusion weighted MRI and extent of hypoperfusion can be deduced by CT. The exact choice, timing and interpretation of these modalities remains within the domain of the art and science of medicine. 


\section{REVIEW}

\section{from page 7}

All the tests have the capability of measuring either anatomical or functional derangement of the brain or indeed both and many have the added capability of depicting pathophysiological events in three axes ( $\mathrm{x}, \mathrm{y}$ and $\mathrm{z}$ axes) and four (time) dimensional domains. In combination with the specialities of cardiovascular medicine and vascular surgery it can readily be appreciated that the heart and the entire cerebrovascular tree can be evaluated noninvasively. This constitutes not only the heart, but also the aortic arch and its major branches, the cervicocephalic vessels (vertebral arteriesVI-V3, common, external and internal carotid), the intracranial carotid (siphon region andV4 vertebral sections) and the circle of Willis and its major branches. Conventional angiography today seems destined largely for the exclusion of infrequent vasculitic disorders and clarification of discrete anatomical features of aneurysms and arteriovenousmalformations.

The current approach to cerebrovascular diagnostics demands an immediate appraisal of the stroke mechanisms as all early and secondary preventative treatment is entirely dependent on determination of the pathophysiology. In the acute stroke situation, the initial three questions the clinician has to answer before initiating treatment are-is it a stroke (and not a neoplasm, seizure or migraine), if it is a stroke, is it bland infarction or haemorrhage; and if bland infarction what subtype (because these have different treatments). Broad categories include 1) small vessel atherosclerotic disease (lacunar), 2) large vessel atherosclerotic disease (carotid, vertebral or intracranial stenoses), 3) cardioembolism (disrhythmias, valvular, dyskinetic segments, paradoxical embolism), 4) other (dissection, prothrombotic, vasculitic) and 5) undetermined groups. Briefly, treatment options include: antiaggregant therapy and risk factor control for small vessel disease, carotid endarterectomy and at times aortic branch vessel bypass for large vessel disease. Most cardiac diseases are eminently treatable: antithrombotics for dissection, anticoagulants for many prothrombotic disorders and immunosuppressive agents for many of the vasculitides constitute effective albeit not always curative treatment today.

Using combinations of the neurodiagnostic armamentarium to advantage is the key to accurate diagnosis and hence cost efficacy. The latter is particularly relevant given current (and very likely future) financial restraints and managed health care. In the clinical context it may be readily appreciated how MRA may clearly depict a stenosis in a large brain supplying vessel which may be graded by transcranial Doppler. Likewise, spiral CT may complement duplex Doppler of a brain supplying cervicocephalic vessel. In both instances invasive intra-arterial angiography is obviated. Transcranial Doppler and duplex Doppler not only rapidly and cost effectively diagnose large artery disease but may also diagnose high intensity transient signals (HITS), diagnostic of emboli in most instances and whether the embologenic site is cardiac, major vessel (carotid bifurcation) or intracranial stenosis. SPECT and TCD have been usefully combined for prognostication and stroke subtype classification, to guide therapy and to deduce a cerebral perfusion index. ${ }^{17}$ Both TCD and SPECT may be used to estimate cerebrovascular reserve using $\mathrm{CO}_{2}$, Acetazolamide or paper bag rebreathing as vasodilatory agents. ${ }^{1}$ Such subtyping of cerebral ischaemic symptomatology allows refinement of treatment options such as earlier and more appropriate carotid endarterectomy (CEA).SPECT iscurrently the best early stroke imaging test and provides objective evidence for diaschisis type neurological deficits, seen with a variety of stroke patients both cortical and subcortical. ${ }^{15}$
As one of many possible illustrative examples, it is easy enough to appreciate that a clinical diagnosis of posterior circulation ischaemia or minor infarction (so called vertebrobasilar insufficiency) is rarely made with confidence. Such patients are notoriously difficult to diagnose and usually have had numerous speciality consultations and a long list of costly nondiagnostic investigations, mainly because they present with a plethora of symptoms that may fluctuate with time. Even isolated tinnitus and vertigo may be presenting features and masquerade as otologic problems, but more commonly a nondescript "wooziness" is reported, much to the despair of the clinician. With a clinical suspicion though, in the absence of other disease, MRA of the vertebral and basilar arteries, usefully combined with transcranial Doppler might detect the responsible vertebral artery origin disease or basilar stenosis. Antiaggregant treatment may be then prescribed with conviction or changed to Ticlopidine or Warfarin as required. Both doctor and patient will benefit from more precise diagnosis of the neurological symptomatology.

Biotechnology has rewarded us with remarkably clear pictures of the brain structure and function that impact on management. The mostchallenging issue facing the clinician and radiologist today is the knowledge of discriminatory use of these modalities. Clearly, no exact step by step flow diagram for stroke investigation can be presented as every stroke patient is different. Investigation must be tailored and individualised. Stroke should be recognised as a multidisciplinary syndrome and so too is the investigation. The way forward seems certain to be a close multidisciplinary liaison for each individual patient. The effective, orderly use of tests can save time, speed up diagnosis and save the patient from undergoing unnecessary, expensive and at times potentially harmful tests. Despite the ever increasing fractionation of medical sub 